\title{
Un groupe d'experts au service de la chimie et de ses industries : Le Comité des arts chimiques de la Société d'encouragement pour l'industrie nationale
}

Gérard EMPTOZ*

\section{Résumé}

Durant les vingt années qui séparent les deux conflits, les seize experts composant le Comité des Arts chimiques de la Société d'encouragement pour l'industrie nationale (SEIN), poursuivent leurs activités au service de la science et de l'industrie chimique. Tout en participant à diverses organisations et manifestations professionnelles mises en place à partir de 1919, ils travaillent à la Société, font des rapports, ou prononcent des conférences sur des sujets d'actualité. Ils poursuivent aussi la politique de remise de récompenses à des individus ou à des entreprises porteurs d'innovations. Avec une répartition professionnelle étendue, composée de savants dont plusieurs membres de l'Académie des sciences, d'ingénieurs et d'entrepreneurs, le Comité réunissait de nombreuses spécialités des secteurs publics et privés.

Les activités que les membres du Comité ont menées durant l'entredeux-guerres permettent de suivre des évolutions scientifiques et techniques dans plusieurs domaines de la chimie, et de déterminer la nature des encouragements qu'ils ont pu donner au service de l'ensemble du secteur.

\section{Mot-clés}

Industrie chimique. Société d'encouragement pour l'industrie nationale. Expertise. Documentation chimique. Innovation. Entre-deuxguerres.

\begin{abstract}
During the interwar period, the sixteen members from the Chemical Art Committee (Comité des Arts chimiques) of the Society for the Advancement of National Industry (Société d'encouragement pour l'industrie nationale, SEIN) continued their work in favour of French science and industry. They not only participated in various organisations and professional groups, created since 1919, but also worked for the Society,

Gérard EMPTOZ, Comité d'histoire de la Société d'encouragement pour l'industrie nationale. Centre François Viète, Université de Nantes. Groupe d'histoire de la chimie - SCF.
\end{abstract}


preparing reports or presenting conferences on contemporary topics. They also continued to award prizes to innovative individuals or companies. In majority composed of professional scientists, the committee included members of the Academy of Sciences, engineers, and entrepreneurs, with a number of experts in different domains drawn from both the public and the private sectors.

Tracing the committee's activities during the interwar period allows us not only to follow the evolutions of science and technology in different areas of chemistry, but also to determine the nature of the support provided by the SEIN to the sector in general.

\section{Keywords}

Industrial chemistry. Société d'encouragement pour l'industrie nationale. Expertise. Chemical documentation. Innovation. Interwar period.

\section{Introduction}

Durant les vingt années qui séparent les deux guerres mondiales, les seize experts qui composaient le Comité des Arts chimiques de la Société d'encouragement pour l'industrie nationale (SEIN), ont poursuivi leurs activités au service de la science et de l'industrie chimique dans la continuité des périodes antérieures. Ils ont ainsi fait des rapports sur des sujets d'actualité en particulier sur les nouvelles technologies, ou prononcé des conférences sur ces questions. Ils ont aussi poursuivi la politique traditionnelle de remise de récompenses à des individus ou à des entreprises porteurs d'innovations.

Composée de savants, dont plusieurs étaient membres de l'Académie des sciences, d'ingénieurs et d'entrepreneurs, le Comité réunissait l'entre-deux des spécialistes dans de nombreux domaines professionnels venus des secteurs public et privé.

En suivant les activités que les membres ont menées durant la guerre, nous pourrons retracer des évolutions scientifiques et 
techniques dans plusieurs domaines de la chimie, et déterminer la nature des encouragements donnés durant cette période de pleine réorganisation du secteur à la sortie du conflit.

\section{Une société au service de l'industrie depuis plus d'un siècle}

Fondée à l'automne 1801 par un groupe de savants, comme Claude Berthollet ou Nicolas Vauquelin, de hauts fonctionnaires, comme Joseph-Marie de Gérando, d'experts techniques, comme Étienne de Montgolfier, d'hommes d'affaires, comme Benjamin Delessert, réunis sous la présidence du chimiste et industriel JeanAntoine Chaptal, alors ministre de l'Intérieur de Napoléon Bonaparte, la Société d'encouragement pour l'industrie nationale (SEIN) a eu pour objectif de « seconder l'industrie dans son développement ». Des encouragements en faveur de l'inventivité et l'activité productive vont constituer l'essentiel des missions que la société a poursuivies sans discontinuité depuis sa fondation et durant les décennies qui ont suivi. De nos jours, elle continue de se manifester dans le soutien à l'innovation.

La société est restée élitiste, ne comptant que quelques centaines de membres, un millier au début du $\mathrm{XX}^{\mathrm{e}}$ siècle, recrutés par cooptation dans les milieux scientifiques et universitaires, les ingénieurs et les chefs d'entreprise, et aussi dans la haute administration et parfois dans les milieux parlementaires. Ils sont en majorité localisés à Paris.

Organisée en comités spécialisés, au nombre de cinq lors de la création: agriculture, arts chimiques, arts mécaniques, arts économiques (au sens des techniques et équipements individuels et 
collectifs), et commerce, la société étendra par la suite son champ d'activité. En 1876, le Comité de construction et beaux-arts est créé, et ce n'est qu'en 1947 que sera créé le Comité de la physique. De nos jours, le Comité de la communication a été adjoint aux précédents.

Pour sa part, le Comité des «arts chimiques » a toujours tenu une place de premier plan dans l'histoire de la société. Dans celle-ci, présidée par Chaptal à la fondation en 1801, ce comité dont le président était membre, a été très actif dès les premières années. Il faut ici souligner le fait que la chimie et ses industries ont été considérées comme un secteur essentiel dans l'économie nationale, en particulier parce que ses activités devaient pénétrer progressivement dans de nombreuses branches industrielles au cours du $\mathrm{XIX}^{\mathrm{e}}$ siècle. De plus les trois premiers présidents, successivement Chaptal, Louis-Jacques Thenard et Jean-Baptiste Dumas, ont tenu cette fonction durant une longue période, la plus longue ayant été celle durant laquelle le chimiste Dumas en a été à la tête, entre 1845 et 1882 .

Avant d'aborder la période de l'entre-deux-guerres, il n'est pas inutile d'évoquer brièvement la période de la première guerre mondiale. Dans une longue lignée d'activités historiques, le Comité a participé largement et a mis ses compétences au service des différentes structures mises en place pour les besoins de la défense nationale. Comme on sait, les besoins industriels ont été énormes et la mobilisation de spécialistes tels que les membres du Comité des arts chimiques a été complète ${ }^{1}$.

\footnotetext{
${ }^{1}$ La période de la guerre de 1914-1918 fait actuellement l'objet de recherches historiques par la Commission d'histoire de la SEIN.
} 


\section{Un groupe de chimistes très actifs pendant la Guerre de 1914- 1918}

Dès la déclaration de guerre, la société prend position avec la réaction immédiate du président de la Société Léon Lindet qui veut mettre les comités au service du pays ${ }^{2}$. Par la suite, ce qui est remarquable, comme on le note dans les comptes rendus des réunions du Comité des arts chimiques, est la participation permanente de Lindet aux activités de ce comité durant le conflit ${ }^{3}$; son soutien a contribué à stimuler les activités de tous ses membres.

On assistera ainsi à la participation du Comité de chimie aux efforts industriels du secteur, notamment à l'Office des produits chimiques et pharmaceutiques, et aussi à d'autres services officiels spécialement organisés par les autorités de l'État.

Il convient ici de mentionner la place tenue au cours du conflit par le président de la SEIN. Léon Lindet était professeur de technologie agricole à l'Institut national agronomique, et, lors de son élection à la présidence, il avait été président pendant plusieurs années des congrès internationaux de chimie ${ }^{4}$. Au cours de sa carrière, il va occuper plusieurs fonctions officielles dont celle de membre du Conseil d'hygiène et de salubrité de la Seine à partir de 1908. Durant la Première guerre mondiale, il entre au Conseil supérieur de l'Agriculture en 1915, au Comité consultatif des arts et manufactures en 1917, et par la suite, au conseil de l'Office national de la propriété

\footnotetext{
${ }^{2}$ Dès le 10 novembre 1914, Léon Lindet fait part au comité de sa rencontre avec Auguste Béhal pour une participation aux activités de l'Office des produits chimiques et pharmaceutiques, voir BSEIN, 114, 150 (1915).

${ }^{3}$ Archives SEIN, Comité des arts chimiques, R02 et R03.

${ }^{4}$ Emptoz Gérard, « Léon Lindet (1857-1929)», Itinéraires de chimistes, 1857-2007, Lestel Laurence (coord.), EDP Sciences, Paris, 2007, p. 345-349.
} 
industrielle, de la Commission supérieure des inventions (délégué de l'Institut), et du Comité consultatif des pétroles.

Par ailleurs, il adhérait à plusieurs sociétés savantes et professionnelles. Membre de la Société chimique de Paris, il faisait partie, dès 1893, de la Société d'encouragement pour l'industrie nationale. Entré dans son conseil d'administration en 1895, et, en 1903, nommé vice-président de la SEIN, il en sera élu président en 1913, et restera à ce poste durant la Première guerre mondiale et après, jusqu'à son décès. Membre de l'Association des chimistes de sucrerie et de distillerie, domaine spécialisé de la chimie industrielle dans lequel il est un expert reconnu, il en est président de 1895 à 1898. En 1899, il est membre de la Société nationale d'agriculture, après avoir été lauréat de cette société en 1891 et en 1896. La chimie ayant donc toujours été au cœur des préoccupations du président Lindet, sa participation active aux activités du Comité des arts chimiques durant la guerre était compréhensible.

Les activités du Comité durant le conflit n'ont pas encore été complètement étudiées, il est donc seulement possible d'en identifier quelques-unes dont le poids apparaît important.

En particulier, sur le plan des produits et des matériels de remplacement qu'il a fallu développer à partir de 1915 pour compenser la disparition de matières premières et de produits chimiques importés d'Allemagne avant la guerre, le président Lindet et les comités, en particulier celui de chimie, ont apporté un soutien aux fabricants de produits et des constructeurs de matériel ${ }^{5}$, avec

\footnotetext{
${ }^{5}$ Des conférences sont organisées à partir de 1915 pour «montrer dans quelles conditions l'industrie française (...) était capable de se reprendre et de lutter » contre la concurrence allemande, $\operatorname{BSEIN~(1915),~p.~532,~et~} B S E I N(1916)$, p. 28 et 34.
} 
l'organisation de deux expositions de matériels et produits, en juin $1916^{6}$ et $1917^{7}$, dans les locaux de l'Hôtel de l'industrie.

Durant la guerre, la société a publié régulièrement des études et des mises au point susceptibles de diffuser l'information scientifique et industrielle, comme l'a souligné son président : «Déjà au cours de la guerre, elle n'a pas manqué de signaler, par l'organe de son Bulletin, par ses cinquante-sept conférences et ses deux expositions, les initiatives prises par nos industriels $»^{8}$.

À l'armistice, le même président lance un appel à tous les membres de la société pour reconstruire et développer les industries dans tous les domaines, en particulier celui des industries de la chimie :

«Notre rubrique: "Les efforts de l'industrie française pendant la guerre " sera maintenue tant que nous n'aurons pas rendu justice à tous ceux qui ont contribué à remplir les vides de notre nomenclature industrielle. Mais, à partir de la signature de l'armistice, leurs efforts ne seront plus catalogués sous cette rubrique: ils seront exposés comme par le passé, dans des notes, des mémoires, des rapports de comités. Il n'y a plus "d'après guerre »; la Paix est un état normal ; la Société reprend ses travaux que la guerre a interrompus $»{ }^{9}$.

\footnotetext{
${ }^{6}$ Lindet Léon, «Les efforts de l'industrie française pendant la guerre », BSEIN (juillet-août 1916), p. 13-36.

${ }^{7}$ Lindet L., « Les efforts de l'industrie française pendant la guerre », BSEIN (juilletaoût 1917), p. 22-58.

${ }^{8}$ « Circulaire adressée aux membres de la Société d'encouragement pour l'industrie nationale par son Conseil d'administration, en date du 20 décembre 1918 », BSEIN (janvier-février 1919), p. 34.

9 "Circulaire adressée aux membres de la Société d'encouragement...(ibid) », BSEIN (janvier-février 1919), p. 34-37.
} 


\section{Une société dans laquelle la chimie est très représentée dans l'entre-deux-guerres}

Pour mesurer l'étendue du réseau scientifique et industriel de la société dans l'entre-deux-guerres, la consultation de l'annuaire de la société permet de mesurer la place de la chimie et de ses industries. La consultation de l'annuaire de la société permet de mesurer la place de la chimie et de ses industries et donc l'étendue de son réseau scientifique et industriel dans l'entre-deux-guerres.

Dans le cas des années 1930, période assez représentative pour la période abordée ici - l'année 1933 sera prise ici comme exemple ${ }^{10}$ -, on constate que les professions de la chimie représentent environ le tiers de l'ensemble des membres de la société ${ }^{11}$. À côté des membres à titre individuel, qui sont des scientifiques, des universitaires, des ingénieurs et des chefs d'entreprise, se trouvent aussi de nombreuses entreprises, ainsi que plusieurs chambres syndicales, des chambres de commerce, et des offices techniques. D'autre part, on note que ce secteur d'activité est bien représenté et qu'il couvre tout le pays. De nombreuses catégories professionnelles sont présentes. Les membres dans les différents secteurs sont répartis ainsi par ordre décroissant: métallurgie (116) ; industries chimiques et pharmaceutiques (90); enseignement et recherche chimique (49) ; cuirs et peaux (46) ; verre, quartz, émaux (32); céramique (22); sucrerie (13); huiles, graisses et savons (12) ; essences et parfumerie (11) ; colles, gélatines, engrais (11) ; poudres et explosifs (10); caoutchouc (10); papeterie (10);

\footnotetext{
${ }^{10}$ Annuaire des membres de la Société d'encouragement pour l'industrie nationale, Paris, 1933 (Arch. SEIN).

${ }^{11}$ Décompte fait dans 1'Annuaire des membres de la Société d'encouragement pour l'industrie nationale, Paris, 1933 (Arch. SEIN).
} 
minoterie et boulangerie (6) ; photographie (6) ; distillerie (6) ; teinture et blanchiment (1); amidonnerie (1); matières plastiques (1).

En comptant la métallurgie comme branche de la chimie, selon les critères de l'époque, l'ensemble des membres « chimistes » de la SEIN comportait 453 membres, soit $42 \%$ des 1076 membres de la société. Si on se limite à la chimie classique et à ses industries, on compte 337 membres, soit $31 \%$ de l'ensemble.

La répartition des activités présentes ici serait à rapprocher de celle qui pourrait être trouvée dans des études des industries chimiques françaises durant cette période historique, tel l'ouvrage de Paul Baud ${ }^{12}$ ou le volume collectif dirigé par Jean Gérard, publié par la revue de la Société de chimie industrielle en $1926^{13}$.

\section{Les membres du Comité des arts chimiques de 1918 à 1939}

Les hommes qui composent le Comité représentent un ensemble de compétences dans des domaines variés du secteur scientifique et industriel représenté. On note que cette composition du Comité est d'ailleurs identique à celle de 1914, ce qui signifie que le groupe a traversé la période de guerre sans changements.

En 1918, le Bulletin présente la composition suivante du Comité :

H. Le Chatelier (président), P. Bérard, A. Carnot, L. Appert, A. Livache, L. Baclé, A. Haller, M. Prud'Homme, L. Guillet, G.

\footnotetext{
${ }^{12}$ Baud Paul, L'industrie chimique en France. Étude historique et géographique, Éd. Masson et C $\mathrm{C}^{\mathrm{ie}}$, Paris, 1932.

${ }^{13}$ Gérard Jean (dir.), 1914-1924. Dix ans d'efforts scientifiques, industriels et coloniaux, Chimie \& industrie, Paris, 1926.
} 
Bertrand, A. Trillat, L. Delloye, J. Loebnitz, H. Gall, A. Pagès, G. Chesneau.

Membres correspondants français : Guimet fils, Pechiney, G. Petitpont, G. Charpy.

Membres correspondants étrangers : E. Solvay (Belgique), R. Hadfield (G-B), H.M. Howe (G-B), A. Legallet (USA?), W.H. Nichols (USA).

En premier lieu, on note la présence de plusieurs ingénieurs polytechniciens : Henri Le Chatelier (membre de l'Institut, École des Mines, Sorbonne), Adolphe Carnot (membre de l'Institut, École des Mines), Léon Baclé (Compagnie des chemins de fer du Nord et forges de Chatillon-Commentry), Maurice Prud'homme (chimiste), Gabriel Chesneau (École des Mines). Plusieurs ingénieurs centraliens : Léon Appert (verreries), Léon Guillet (École centrale de Paris (ECP) et CNAM), Lucien Delloye (Saint-Gobain); des chimistes et ingénieurs chimistes : Paul Bérard (Sorbonne), Auguste Trillat (Institut Pasteur), Achille Livache, (département de la Seine) ; des pharmaciens : Albin Haller (membre de l'Institut, Sorbonne), Gabriel Bertrand, (Institut Pasteur et Sorbonne) sont présents, ainsi que des entrepreneurs : Jules Loebnitz (faïencerie), Henri Gall (électrochimie), Albert Pagès (Syndicat des produits chimiques). On note aussi la présence de Daniel Berthelot (pharmacien), professeur à l'École de pharmacie de Paris, comme membre du Comité des arts économiques. On peut aussi ajouter que Lindet est pour sa part membre du Comité d'agriculture.

\section{Un Comité profondément remanié au cours des décennies suivantes}

Lorsqu'on se reporte à la fin des années 1930, la composition du Comité apparaît bien différente, en particulier à la suite de plusieurs décès survenus durant les années 1920 et 1930. Ces 
disparitions ont entraîné la cooptation de membres représentant de nouvelles compétences. Ainsi sur l'ensemble des seize membres statutaires, on compte douze nouveaux venus, ce qui signifie un renouvellement en profondeur, soit les trois-quarts du Comité, en l'espace de vingt années. Certaines évolutions du domaine de la chimie intervenues durant cette période historique se font jour.

Ainsi, en 1939, le Bulletin de la société présente la composition suivante du Comité :

L. Guillet (président), G. Bertrand, A. Trillat, J. Loebnitz ,G. Charpy, A. Portevin, P. Pascal, A. Wahl, P. Jolibois, R. Dubrisay, H. Jossier, G. Painvin, E. Damour, R. Berr, M. Javillier, E. Fleurent, G. Claude.

Membres correspondants : L. Zuber, J. Durand, G. Chaudron. Membres correspondants étrangers: Sir R. Hadfield, E. Hauser, A. Sauveur, L. Mrazec, Sir H. Cort, E. Votocek, G. Longinescu. Au Comité des arts économiques : L. Pineau.

Les origines des nouveaux membres se répartissaient selon une composition proche de celle de 1918. Autour d'un président centralien, Léon Guillet (directeur de l'École Centrale de Paris), on trouve plusieurs polytechniciens: Georges Charpy (membre de l'Institut), Pierre Jolibois (École des Mines de Paris), René Dubrisay (Manufactures des Tabacs), Georges Painvin (société Ugine), Raymond Berr ${ }^{14}$ (société Kuhlmann), et naturellement des centraliens: Albert Portevin (membre de l'Institut, ECP), Henri Jossier (cuirs et peaux). Les autres formations sont toujours représentées par un pharmacien : Maurice Javillier (Institut Pasteur, Sorbonne et CNAM), des chimistes et ingénieurs chimistes : Paul

\footnotetext{
${ }^{14}$ Au sujet de Raymond Berr et d'André Wahl, la Société aura à déplorer la perte de ces deux membres éminents victimes en 1944 de la barbarie antisémite.
} 
Pascal (Sorbonne); André Wahl (CNAM), Emilio Damour (CNAM), Émile Fleurent (ESPCI, CNAM), Georges Claude (ESPCI, Air liquide). L'émergence d'ingénieurs issus de l'ESPCI est manifeste, et ce constat peut être fait par ailleurs au sein des divers comités officiels créés durant la guerre pour les fabrications chimiques. C'est une nouvelle génération d'ingénieurs-chimistes qui est arrivée aussi à la SEIN.

On peut aussi noter la présence de Louis Pineau (Saint-Cyr), directeur de l'Office national des combustibles liquides, entré au Comité des Arts économiques, pour représenter un nouveau secteur clé de la chimie durant l'entre-deux-guerres.

Finalement, il apparaît que le secteur d'activité le plus représenté est encore celui de la métallurgie, avec même son renforcement par rapport à la période précédente. On verra plus loin que les activités identifiées dans le Bulletin confirment une participation très active de ce groupe de spécialistes.

\section{Les activités du Comité vues à partir du Bulletin de la société}

Les articles parus entre 1919 et 1939 constituent des sources essentielles pour connaître les activités du Comité. Toutefois, le nombre de pages imprimées du Bulletin a diminué par rapport à la période d'avant-guerre, en particulier pour des raisons financières. De plus les études spécialisés et les panoramas par secteurs sont moins nombreux qu'auparavant. On trouve surtout des rapports au nom du Comité des arts chimiques sur les travaux d'un spécialiste, de ses 
recherches ou d'un de ses ouvrages, ou d'un entreprise plutôt que des études originales ${ }^{15}$.

De plus, cette période est marquée par la création de la Société de chimie industrielle en 1917, suivie du lancement de sa revue Chimie et industrie, ce qui a modifié de manière très significative le paysage de la chimie vue sous l'angle industriel qui était une spécialité en France de la Société d'encouragement jusqu'à la guerre. Cette nouvelle organisation concernait aussi les moyens de diffusion de l'information dans les domaines des industries chimiques. On reviendra plus loin sur ce point.

\section{La variété des sujets traités dans le Bulletin de la société}

Durant les vingt années de l'entre-deux-guerres, un ensemble conséquent de publications relevant de la chimie et de la métallurgie a paru dans le Bulletin de la société. Durant les années 1920 (19191929), ce sont 59 rapports, recensions et conférences ${ }^{16}$. Dans la décennie suivante (1930-1940), on dénombre 64 rapports, recensions et conférences. Tout en constatant l'étendue des sujets traités durant ces deux décennies, il est possible de donner de manière succincte les axes principaux des travaux du Comité. Trois domaines sont dominants : la métallurgie, les engrais, et les carburants. Et des auteurs sont particulièrement actifs : Albert Portevin, Léon Guillet,

\footnotetext{
${ }^{15}$ Les membres du Comité effectuent ainsi une veille scientifique et technologique.

${ }^{16}$ La présente enquête sur les publications de la Société a été réalisée par la consultation de la base informatisée que la Commission d'histoire a créée à partir de la compilation des tables annuelles du Bulletin. Cette base est actuellement accessible dans les locaux de la société.
} 
René Dubrisay, Auguste Trillat, Albin Haller, Louis Baclé, Henri Le Chatelier, et Gabriel Bertrand.

$\mathrm{Au}$ préalable, on note que, naturellement, des bilans sont dressés à la sortie du conflit, mais leur nombre reste limité. Ainsi en 1919, L. Guillet fait le point sur «les industries métallurgiques à l'avant-guerre et leur avenir $»{ }^{17}$. En 1920, A. Haller décrit les efforts de «l'industrie chimique française pendant la guerre: matières premières servant à la préparation des poudres et explosifs ${ }^{18}$ pour produire les matières explosives de toute nature (deux conférences). La même année, L. Guillet décrit les travaux et essais effectués de 1915 à 1918 aux laboratoires de l'aéronautique de Chalet-Meudon ${ }^{19}$.

C'est en 1923 que la société publie aussi le «Document confidentiel allemand» sur les industries françaises, étude détaillée qui soulignait les forces et les faiblesses de notre pays durant la guerre $^{20}$.

\section{La métallurgie : un domaine très bien suivi et soutenu}

Les auteurs des principales contributions ont été des spécialistes reconnus, comme en premier lieu Portevin (avec 19 articles), Guillet (18 articles), et plus modestement Baclé et H. Le Chatelier (6 articles chacun). Les grandes lignes durant les années 1920 portent sur les sujets décrits ci-après.

\footnotetext{
${ }^{17} \operatorname{BSEIN~(1919),~p.~} 429$ sq.

${ }^{18}$ BSEIN (1920), p. 382-384 et 384-386.

${ }^{19} \operatorname{BSEIN~(1920),~p.157~sq.~}$

${ }^{20}$ BSEIN (1923), p. 586-640.
} 
1) Sur la métallurgie du fer. Les progrès dans la métallurgie du fer, méthodes d'analyse et d'essais, rôle des laboratoires dans l'industrie, avec des mises au point, des conférences, des recensions, des rapports sur des travaux signalés pour des récompenses attribuées par la société ${ }^{21}$.

2) Sur la métallurgie des métaux légers. En ce domaine, un effort particulier est manifeste, principalement pour mettre en valeur la métallurgie de l'aluminium, métal léger dont les emplois sont encore à l'étude dans l'entre-deux-guerres. Une mise en perspective des travaux réalisés par les membres de la SEIN avec ceux que les recherches récentes sur l'histoire de l'aluminium en France ont mis en lumière permettrait de mesurer comment les initiatives se sont articulées $^{22}$.

Une grande manifestation sur «Des nouvelles applications industrielles de l'aluminium, du magnésium, du calcium et du sodium » (21 au 28 mai 1921), est marquée par les interventions durant la semaine concernée de: L. Guillet, sur l'industrie de l'aluminium ${ }^{23}$; E. Dusaugey et Zetter, sur les emplois de l'aluminium dans 1'électricité24 ; G. Flusin, sur le magnésium, le calcium et le

\footnotetext{
${ }^{21}$ Un bilan peut être dressé à partir des tables annuelles du BSEIN, entre 1920 et 1940, sur le site du CNUM. Les auteurs cités ci-dessus ont fréquemment fait des rapports écrits sur les recherches en cours.

${ }^{22}$ Voir : Hachez-Leroy Florence, «L'Aluminium français. L'invention d'un marché. 1911-1983 », Annales. Histoire, Sciences Sociales, 56/6, 1398-1400 (2001).

${ }^{23}$ Guillet Léon, «Conférence sur l'aluminium, sa fabrication, ses propriétés, ses alliages, leurs emplois », BSEIN (1921), p. 683-736.

${ }^{24}$ Dusaugey Ernest, «L 'emploi de l'aluminium dans l'électricité », BSEIN (1921), p. 763-786; Zetter Charles, «Les emplois de l'aluminium dans l'appareillage électrique », BSEIN (1921), p. 919-956.
} 
sodium $^{25}$; MM.Trillat et Guérin, sur les emplois de l'aluminium dans les industries alimentaires et chimiques ${ }^{26}$; Lt-Col. Grard, les alliages légers en aéronautique ${ }^{27}$; R. de Fleury, les métaux légers en mécanique et automobile ${ }^{28}$.

Enfin, l'Exposition, organisée à l'occasion de ces conférences dans les locaux de l'Hôtel de l'industrie, a été visitée par «plus de 5000 personnes $»$.

\section{L'émergence de nouvelles technologies : les carburants et les engrais}

Les premiers articles parus en janvier 1920 donnent la place à des sujets d'actualité qui représentent les préoccupations principales du gouvernement de l'époque, face à des manques de ressources. La question du pétrole se pose devant le développement des transports automobiles, et la dépendance vis-à-vis de l'étranger, en particulier des États-Unis. La question de l'agriculture qui se trouve confrontée à un manque de main-d'œuvre à la fin de la guerre et à une production agricole insuffisante qu'il faut moderniser. On peut citer sur ces deux thèmes plusieurs articles publiés par la SEIN dans son Bulletin.

Sur la synthèse de l'ammoniac, sujet d'actualité durant la guerre pour les matières explosives et, avec le retour à la paix, pour

\footnotetext{
${ }^{25}$ Flusin Georges, «Le magnésium, le calcium et le sodium », BSEIN (1921), p. $788-812$.

${ }^{26}$ Trillat Auguste, «Sur 1'emploi de l'aluminium dans les industries de la fermentation (brasseries, etc.), en laiterie, en fromagerie, etc. », BSEIN (1921), p. 813-840.

${ }^{27}$ Grard Charles (Lt-Col.), « Les alliages légers et leur emploi en aéronautique », BSEIN (1921), p. 864-893.

${ }^{28}$ Fleury Raymond de, «Les métaux légers dans la construction mécanique et, en particulier, dans l'industrie automobile », BSEIN (1921), p. 896-918.
} 
les engrais, Marcel Guichard et ses collègues, présentent les essais réalisés sous le titre : "Contribution à l'étude de la synthèse directe de l'ammoniac par catalyse sous pression $»^{29}$.

Sur le pétrole, dont notre pays a toujours été dépendant, et que la restitution de l'Alsace laisse espérer apporter un soulagement limité, la conférence de Paul de Chambrier: «Les gisements de pétrole d'Alsace » permet de mesurer l'importance stratégique de ces gisements pétroliers et la nature des équipements techniques de leur exploitation $^{30}$.

En réponse à une demande gouvernementale, un domaine majeur est mis en avant, celui des combustibles liquides et solides, notamment avec la description des travaux de la Commission interministérielle de la carbonisation ${ }^{31}$. Au cours des années suivantes, les lecteurs sont tenus informés très régulièrement sur les travaux de cette commission ${ }^{32}$.

La question du «carburant national » apparaît en 1923, au moment où se met en place la politique de l'État pour encourager les recherches destinées à pouvoir faire fonctionner les moteurs à explosion par des succédanés. Le sujet est crucial devant le manque d'approvisionnement régulier en pétrole dont souffre le pays. Le décret portant sur la proportion d'alcool dénaturé qui peut être

\footnotetext{
${ }^{29} \operatorname{BSEIN~(1920),~p.~71-102.~}$

${ }^{30}$ BSEIN (1920), p. 45-70. Le site pétrolier alsacien de Pechelbronn, revenu dans le territoire national après la fin du conflit, est donc un lieu de production de très grande importance dans les années 1920.

${ }^{31} B S E I N(1920)$, p. 241.

${ }^{32}$ BSEIN (1921), p. 126-136; 286-296; 478-508. Ces articles se poursuivent en 1922.
} 
mélangé au carburant automobile est reproduit dans le Bulletin $^{33}$, après la reproduction du décret du 30 mai 1923 sur l'importation en France des essences.

Une autre voie est celle de la carbonisation, par exemple à basse température en particulier des lignites $^{34}$, et celle d'autres matériaux (bois notamment) qui seront abordées au cours de la période.

\section{Des questions d'avenir en discussion}

En ce domaine, plusieurs articles sont autant techniques que politiques. On peut citer ici, dans le thème des problèmes d'énergie posés dans l'après-guerre, l'article de Albert Guiselin, «Comment nous libérer du pétrole étranger ? ${ }^{35}$. Il s'agit de la conférence faite à la société par un ingénieur-chimiste, membre du Comité général du pétrole, qui passe en revue tous les problèmes posés par la pénurie de pétrole et les solutions qui ont déjà été étudiées ou qu'il faudrait étudier pour surmonter les difficultés. Il veut montrer que la France et ses colonies peuvent fournir des substituts du pétrole. Sont abordés successivement les lubrifiants, les pétroles lampants puis les combustibles liquides. Pour souligner qu'en faisant appel aux ressources nationales, par carbonisation de schistes, des lignites, des tourbes, des bois, ou des produits de fermentation alcoolique, ou des produits issus de la carbochimie, de synthèses organiques ou de gaz pauvres, il est possible de trouver des solutions.

\footnotetext{
${ }^{33}$ BSEIN (1923), p. 448-452.

${ }^{34}$ BSEIN (1924), p. 44-66.

${ }^{35}$ BSEIN (1924), p. 576-601.
} 
Abordant ensuite la question de «comment se libérer du pétrole étranger », l'auteur propose deux solutions : brûler mieux en économisant la ressource, et produire des succédanés d'origine française, ce qui implique « la nécessité d'une alliance entre la science et le capital». Cet article est complété de nombreuses informations techniques et économiques sur les productions, les tonnages et les prix des différents substituts du pétrole.

La même année, l'industrie du gaz fait à son tour l'objet d'articles donnant un panorama très complet. On notera ceux-ci : «L'avenir de l'industrie gazière: consommation, procédés de fabrication, traités de concession, prix de revient futur du gaz ${ }^{36}$, et «L'état actuel de l'industrie gazière ${ }^{37}$.

\section{Un domaine passé au second plan : les colorants et l'absence des polymères}

Alors qu'il a constitué aux yeux des chimistes le domaine essentiel de préoccupation et de développement industriel avant la guerre, les colorants artificiels se semblent susciter maintenant pour la SEIN qu'un intérêt moindre. Sur le plan économique, la réorganisation des industries chimiques a notamment déplacé l'intérêt des spécialistes vers d'autres domaines : chimie des gaz, carburants, chimie des polymères notamment. D'autre part, pour la diffusion des informations industrielles les concernant, les colorants ont des revues spécialisées. De son côté, le Bulletin est devenue une revue plus généraliste qu'auparavant. Par ailleurs, la jeune revue Chimie \&

\footnotetext{
${ }^{36} \operatorname{BSEIN~(1921),~p.~602-629.~}$

${ }^{37}$ BSEIN (1921), p. 630-638.
} 
Industrie ouvre largement ses rubriques aux colorants. Aussi, une seule mise au point marquante est notée pour la période, avec les conférences d'André Wahl : « Les progrès réalisés dans l'industrie des matières colorantes depuis $1914 »^{38}$. Finalement, on relève quelques articles en nombre limité dans le Bulletin sur le sujet.

Enfin, le domaine des fibres artificielles, secteur industriel en plein essor en région lyonnaise notamment, semble absent des articles du Bulletin. Les fibres artificielles, notamment l'acétate de cellulose, constituent un domaine important, mais les recherches engagées dans plusieurs pays étrangers (États-Unis, Grande-Bretagne, Allemagne et URSS principalement) sur les fibres synthétiques ne donnent pas lieu à des mentions dans le Bulletin. C'est sans doute la revue Chimie \& Industrie qui le fera ${ }^{39}$. Toutefois, on trouve mention d'un article sur les matières plastiques en 1935 et une «exposition des applications artistiques françaises de l'industrie des matières plastiques » en $1939^{40}$.

\section{Les conférences destinées au grand public}

Plusieurs axes peuvent être distingués à ce sujet: la publication dans le Bulletin des conférences invitées, ponctuelles ou

\footnotetext{
${ }^{38} B \operatorname{SEIN}$ (1933), p. 146-152.

${ }^{39} \mathrm{Au}$ sujet des recherches sur les fibres synthétiques, voir : Fred Aftalion, Histoire de la chimie, Paris, Masson (1997) ; Jean-Marie Michel, L'histoire industrielle des polymères en France, SCF http://www.societechimiquedefrance.fr/1/documentationsscientifiques/contribution-a-l-histoire-industrielle-des-polymeres-en-france-parjean-marie-michel/

${ }^{40} B \operatorname{SEIN}$ (1939), p. 341-345.
} 
régulières, et des conférences extérieures; le soutien à des initiatives externes à la société (c'est une tradition de la société); la création des conférences Carrion ${ }^{41}$ (1935): la biologie et ses applications industrielles entrent dans la société ; la diffusion dans le Bulletin de ces manifestations permet de conserver le lectorat, de l'élargir vers des problèmes économiques et sociaux.

La publication des conférences devient une activité régulière du Bulletin. L'exemple des conférences de Raymond Berr avec les articles suivants en sont des témoins : «L'évolution de l'industrie des engrais chimiques ${ }^{42}$; «La technique chimique devant la crise $^{43}$; «Essor de la chimie, de Kuhlmann à Berthelot » ${ }^{44}$.

\section{Les évolutions des moyens de diffusion de l'information scientifique et technique en chimie et leurs répercussions au sein de la SEIN}

En ce domaine, comme cela a été signalé plus haut, la création de la Société de chimie industrielle (SCI) en $1917^{45}$ et de la revue Chimie \& industrie en 1918, constitue un changement majeur pour la circulation de l'information et de la documentation au sein de la

\footnotetext{
${ }^{41}$ La première conférence de biologie appliquée organisée à la suite du legs du pharmacien Henri Carrion a été inaugurée le 23 mars 1935, voir BSEIN (1935), p. 323-333.

${ }^{42}$ Conférence au CNAM, mai 1930, BSEIN (juin 1930), p. 483-515.

${ }^{43}$ Conférence au XIII ${ }^{\mathrm{e}}$ congrès de la Société de chimie industrielle, septembre 1933, BSEIN (novembre 1933), p. 565-572.

${ }^{44}$ Conférence à la Société des ingénieurs civils, janvier 1939, BSEIN (mars 1939), p. 152-174.

${ }^{45}$ Fell Ulrike, «Quelle liaison entre science et industrie ? La Société de chimie industrielle entre les deux guerres, 1917-1939», Chimie et industrie en Europe. L'apport des sociétés savantes industrielles du XIXe siècle, Fell U. (dir), Éditions des Archives contemporaines, Paris, 2001, p. 69-95.
} 
chimie française ${ }^{46}$. La Société d'encouragement n'est pas étrangère à ces initiatives. En effet il apparaît que plusieurs membres en particulier ceux du Comité de chimie ont participé à la mise en place de cette nouvelle structure associative. Ainsi on retrouve dans l'organisation de la SCI à sa fondation en 1917, les noms d'Albin Haller et de Henri Le Chatelier, comme présidents d'honneur, d'Adolphe Carnot, de Léon Lindet alors président de la SEIN et d'Ernest Solvay, membre associé du Comité de chimie, parmi les membres d'honneur. La moitié des membres d'honneur sont donc aussi des membres de la SEIN. Parmi les membres du Conseil d'administration, placé sous la présidence de l'industriel Paul Kestner, on compte cinq membres ou futurs membres du Comité de chimie sur les 58 membres : Daniel Berthelot, Georges Charpy, Georges Claude, Albert Pagès, André Wahl, sans compter d'autres membres ne faisant pas partie du Conseil d'administration de la SEIN.

Plusieurs membres du Comité des arts chimiques ont été aussi membres du «Comité de rédaction des publications» de la revue Chimie \& Industrie à sa création: tels Gabriel Bertrand, Adolphe Carnot, Georges Charpy, Gabriel Chesneau, Georges Claude, Émile Fleurent, Albin Haller, Pierre Jolibois, Paul Pascal, Albert Portevin et André $\mathrm{Wahl}^{47}$.

\footnotetext{
${ }^{46}$ Fauque Danielle et Emptoz Gérard, «Les chimistes, leurs institutions et leurs sociétés savantes entre les deux guerres. Introduction », L'Actualité chimique, 378379, 120-123 (octobre-novembre 2013); Fauque D., «Les chimistes, leurs institutions et leurs sociétés savantes entre les deux guerres ", L'Actualité chimique, 380, 39-44 (décembre 2013). Voir aussi : https://fr.wikipedia.org/wiki/Soci\%C3\%A9t\%C3\%A9 de chimie industrielle

47 «La Société de chimie industrielle, son organisation», Chimie \& Industrie (décembre 1917), p.12-14, plaquette d'information in Chimie \& Industrie, 1/1 (juin 1918).
} 
Comme la jeune société a bénéficié du soutien des chimistes qui participaient à l'effort de guerre, il est clair qu'elle a trouvé, en particulier, des personnalités de premier plan dans le Comité des arts chimiques de la SEIN. Comme cela avait été le cas pour la Société chimique de Paris $^{48}$ à ses débuts en 1857 et d'autres ${ }^{49}$, la SEIN ne manqua pas d'apporter son appui à la nouvelle société.

Le changement significatif pour les lecteurs qui venaient régulièrement à la SEIN pour se documenter se situe lors du déménagement de la Société chimique de France, qui était installée depuis 1859 comme locataire de la Société d'encouragement, au 44 rue de Rennes. Elle s'installe alors à la Maison de la Chimie, ouverte en 1934, au 28 rue Saint-Dominique. Ce départ va avoir des répercussions sur les collections de chimie que l'immeuble de la Société d'encouragement conservait sur place, à savoir les siennes et aussi celles de la Société chimique. La gestion des deux collections ayant été commune jusqu'à cette date, l'intérêt pour les lecteurs était de trouver dans le même lieu un ensemble très important d'ouvrages et de périodiques français et étrangers en chimie générale et en chimie industrielle.

La fondation de la Maison de la chimie en 1927 et ses conséquences va donc modifier cette situation ${ }^{50}$. Celle-ci va accueillir

\footnotetext{
${ }^{48}$ Celle-ci est restée au 44 rue de Rennes jusqu'à son départ au 28 rue SaintDominique en 1934. Par la suite, la Société rejoindra l'immeuble de la rue SaintJacques.

${ }^{49}$ Benoit Serge, «La Société d'encouragement, pôle majeur de la sociabilité scientifique, technique et industrielle », Encourager l'innovation en France et en Europe, Benoit Serge, Emptoz Gérard et Woronoff Denis, (éd.), éd. CTHS, Paris, 2006, p. 95-120.

${ }^{50}$ Voir à ce sujet Fauque D., « La documentation au cœur de la réorganisation de la chimie dans l'entre-deux-guerres: Rôle des sociétés savantes et institutions
} 
dans un premier temps la SCI et son office de documentation chimique. Le projet très ambitieux qui a pris place vise à la création d'un Centre de documentation chimique dont la guerre avait montré cruellement l'absence. Le départ de la Société chimique est donc accompagné de celui de ses collections vers la rue Saint-Dominique. En 1934, suite à cette installation, la bibliothèque de la Maison de la chimie va alors réunir les collections de la SCI, de la SCF, de la Société de chimie physique, de la Société de chimie biologique et celle de l'Association des chimistes de sucrerie et de distillerie, constituant ainsi un ensemble remarquable de périodiques et d'ouvrages.

Peu avant ce départ des collections de la SCF, la SEIN avait connu un autre événement touchant la gestion des collections avec le départ en 1920 du chimiste Jules Garçon qui était le responsable de la bibliothèque depuis $1904^{51}$. Ce spécialiste connu pour ses mises à jour bibliographiques de chimie était responsable des bibliothèques de la SEIN et de la SCF. Son départ, suivi quinze ans plus tard du déménagement de la SCF et de ses collections, va donc contribuer à réduire progressivement les mises à jour bibliographiques très utiles aux chimistes, et, en parallèle, entraîner la réduction du budget alloué aux abonnements de périodiques dans une période difficile sur le plan financier. On peut dire qu'au milieu des années 1930 les lecteurs

françaises dans le contexte international », Revue d'Histoire des Sciences, 69/1 (janvier-juin 2016), 41-75. L'information reste limitée à ce sujet sur le site: https://fr.wikipedia.org/wiki/Maison_de_la_Chimie.

${ }^{51}$ Blouin Daniel, «Historique de la bibliothèque de la Société d'encouragement pour l'industrie nationale », novembre 2014 :

https://histoiresein.hypotheses.org/686. 
chimistes vont progressivement perdre l'habitude de venir faire leur bibliographie à la SEIN.

Par ailleurs, les célèbres Chemical Abstracts, que l'American Chemical Society a publiés à partir de $1907^{52}$ pour la diffusion en langue anglaise de résumés de la plupart des articles parus dans des périodiques de chimie au niveau mondial ${ }^{53}$, sont entrés dans la bibliothèque de la SEIN en $1913^{54}$. Bien informé, Jules Garçon a dû peut-être se sentir concurrencé par cette publication américaine qui couvrait tous les domaines de la chimie scientifique et industrielle au niveau international.

Finalement, à la fin des années 1930, les collections de chimie de la Bibliothèque de la SEIN, qui étaient jusque-là composées par un grand nombre de revues de chimie industrielle françaises et étrangères, ont diminué progressivement, voire ont été interrompues, affaiblissant ainsi la place historique qu'elles avaient tenue au cœur de la capitale.

\section{Un fonctionnement de la société qui est marqué par des évolutions}

La lecture du Bulletin permet de noter quelques évolutions significatives sur cette période historique. Des médailles, encore nombreuses, sont attribuées régulièrement à des travaux terminés et

52 Chemical Abstracts Service, «About CAS, CAS 100th Anniversary, CAS History: Milestones » (2009)» :

http://www.cas.org/aboutcas/cas100/annivhistory.html.

${ }^{53}$ Ces résumés en anglais viennent concurrencer une littérature similaire, publiée en langue allemande, qui était omniprésente et incontournable à cette époque pour la documentation des chimistes.

54 Benoit S. et Emptoz G., «Les collections de chimie de la Société d'encouragement pour l'industrie nationale », Revue d'Histoire des sciences, 69/1, (janvier-juin2016), 137-151. 
des entreprises ayant réussi, mais il n'y a plus de concours. On peut noter aussi que la chimie est encore assez bien représentée au sein de la société, comme cela apparaît avec les médailles et prix décernés en 1922 et 1923, au cours des manifestations de la célébration du centenaire de l'utilité publique de la SEIN. C'est d'ailleurs une tendance de la société de remettre durant ces décennies de l'entredeux-guerres un grand nombre de médailles...

On note le suivi des principales publications françaises, mais l'absence de bibliographies internationales. Il convient ici de revenir sur l'histoire de la bibliothèque de la société mentionnée plus haut. Jusqu'en 1920, Jules Garçon avait tenu ce service de manière très active depuis près de vingt ans. Après sa démission, ce spécialiste des bibliographies internationales en chimie n'a pas réellement été remplacé. De plus, les ressources financières affectées par la société aux périodiques ont été réduites. Il était alors normal qu'à terme, les comptes-rendus d'articles soient devenus plus rares.

Sur le plan des spécialités, on constate un repositionnement dans les réseaux de la chimie et de la métallurgie, face à d'autres institutions. La Société de chimie industrielle, encore jeune, n'est pas la seule à diffuser l'information scientifique et technique comme on l'a vu. Comme le montre Danielle Fauque, les institutions internationales de chimie contribuent à accroitre fortement les échanges et à disséminer l'information chimique ${ }^{55}$. En France, on peut ajouter que pour encourager l'innovation, on verra la création de l'Office national des recherches scientifiques et industrielles et des

\footnotetext{
${ }^{55}$ Fauque D., « La documentation au cœur de la réorganisation de la chimie », op. cit., note 50 .
} 
inventions en $1922^{56}$. Une société, comme la Société des savants et inventeurs de France (1924), pouvait contribuer aussi à ce mouvement.

\section{L'esquisse d'un bilan pour la période}

La Seconde guerre mondiale va apporter d'autres changements dans l'organisation de la SEIN. Les comités existants avant le conflit sont inchangés et voient aussi l'arrivée d'un nouveau comité, celui des arts physiques en 1948. Chimie et physique pourront ainsi se côtoyer et échanger dans les mêmes lieux.

Pour sa part, le Comité des arts chimiques va continuer de jouer un rôle reconnu, et s'adapter au nouvel environnement mis en place après la Libération. La Bibliothèque va rouvrir vers 1947. Elle aurait pu redevenir « un pôle actif de documentation si une politique d'achats d'ouvrages et d'abonnements avait pu être mise en place par un personnel spécialisé ${ }^{57}$. Le Bulletin, dont la parution a été arrêtée en 1943, ne reprend pas et est remplacé en 1948 par une nouvelle publication, intitulée L'industrie nationale. Toutefois, celle-ci ne retrouvera pas l'importance qu'avait eue le Bulletin. En particulier, les articles se font rares en chimie, et les notes de lecture ont rapidement disparu à la fin des années 1940. Plus généralement, il semble que le

\footnotetext{
${ }^{56}$ Galvez-Béhar Gabriel, «Le savant, l'inventeur et le politique le rôle du soussecrétariat d'État aux inventions durant la première guerre mondiale », $X X^{e}$ siècle, Revue d'histoire, 85, 184-203 (2005) ; Ib., "The "French connection": French Scientists and International Debates on Scientific Property during the Interwar Period », (2013) : https://halshs.archives-ouvertes.fr/halshs-00839580

${ }^{57}$ Blouin D., « La bibliothèque de la Société d'encouragement : un projet logique, une histoire complexe », note de présentation (2015), https://histoiresein.hypotheses.org/686.
} 
Comité de chimie se manifeste bien moins que dans toutes les périodes antérieures. Ses activités se poursuivent, mais il n'en reste pas de traces imprimées comme auparavant. Il devient alors difficile de reconstituer ces activités, et seul un travail dans les archives de la SEIN pourra permettre de suivre les évolutions durant cette période de l'après-guerre. De plus l'étude faite dans le présent article n'est pas exhaustive, et devra être approfondie ultérieurement, compte tenu de l'abondance des articles parus dans le Bulletin de la société durant la période considérée ici.

Parallèlement, après la Seconde guerre mondiale, le panorama des institutions de recherche et des industries d'État concernées par la chimie et ses applications devient très étendu. Des organismes de recherche comme le CNRS, le CEA, l'ONERA, ou l'INRA, réunissent comme experts des scientifiques et des ingénieurs, mettent en place leurs propres centres de documentation et organisent des veilles scientifiques et technologiques pour leurs propres besoins. Devant cette nouvelle situation, la SEIN perd progressivement la place centrale qu'elle avait tenue au service de l'invention et de l'innovation durant un siècle et demi. De nos jours, la SEIN, toujours active, est en train de retrouver une place dans le paysage scientifique et industriel français, et le Comité des arts chimiques y participe activement.

Pour conclure, nous pourrions dire que, durant l'entre-deuxguerres, le Comité des arts chimiques semble avoir été un laboratoire d'idées (think tank) plus qu'un initiateur d'innovations en chimie. 